\title{
Removing the Incarcerated Tunneled Cuffed Venous Catheters: An Experience from a Single Center
}

\author{
Li Zhou Shaobin Yu Shenju Gou Min Shi Tianlei Cui Ping Fu \\ Division of Nephrology, Kidney Research Institute, West China Hospital, Sichuan University, Chengdu, China
}

To the Editor

Central venous catheters (tCVCs) are used in hemodialysis patients. Usually tCVCs can be removed by dragging. However, in the event of the catheter getting stuck, then removing it becomes difficult or even impossible sometimes. The occurrence rate of stuck tCVCs is $1.5-19 \%$ [1-4]. The pathogeneses of tCVCs incarceration are complicated, including the cumulative indwelling time as the most important risk factor [2], vessel calcification, repeated catheter-related infections, vascular curvature of cannulation site, and the fibrin sheath of tCVCs.

Although the methods of removing the stuck catheter had been documented to a great extent, most of the studies were only case reports, including routinely an angioplasty balloon [5], or a laser sheath [6]; or stiff guidewire as included to apply a "pushin" force [7] combined with an introducer sheath and rotator [8]. Actually, all these reported methods were relatively complex with the use of an extra device. Some were even resulting in serious complications such as pulmonary embolism, cardiac perforation, and angiorrhexis.

Here we introduced a novel stretch technique to remove incarcerated tCVCs;

S.Y. and L.Z. contributed equally to this work. this technique was simple, safe, easy to follow, without any extra device, and economical too. The efficacy and safety of this new technique had been investigated in 20 hemodialysis patients retrospectively in our hospital (mean duration time of catheters was 5.65 years [2.1-10.2 years], made by poliurethan, tip-to-cuff as 19 or $29 \mathrm{~cm}$ long). It was observed that these patients had occlusions of the right internal jugular, right innominate vein or the junction, or it was found that the tips of catheters were split or adherent to the vein wall. Under the guide of DSA (digital subtraction angiography), the stretch technique was used skillfully. Balloon dilation was used only in the event of the stretch technique failing.

In detail, after the Dacron cuff was separated, the elasticity of the tCVC was used by stretching it to the maximum extent (there is no elastic felling any more) to fasten the removal. Under the guide of DSA, we kept the angle between the catheter and skin as $30^{\circ}$ and dragged the catheter out several times ( $\leq 20$ times). In these 20 patients, 17 were successfully treated without any extra devices ( $6.3 \pm 3.1$ times $)$. New catheters were then inserted by using the routing cannulation method (14/17). When the routine cannulation method did not work due to the occlusion of the central vein, we catheterized a tCVC by punctur- ing the superior vena cava directly under the guide of DSA (3/17) [9]. Fracture and wreckage of catheters or fatal complications were not found intraoperative. Only in $3(3 / 20)$ patients, it was not possible to pull the catheter out even after stretching for 20 times; later, tCVCs were removed using the balloon dilation technique.

Although this technique was simple, more attention should be paid for preventing the central vein rupture or breakage/rupture of catheter. If the frequency of the pulling catheter was consistent with the heart beats, then it is an indication that the catheter was surrounded by the calcified fibrin sheath or compressed by the right innominate vein [10]. Balloon dilation, thoracic surgery, or embedding in situ should be used instead of pulling the catheter when the mediastinum followed the pulled catheter up and down [10]. Other suggestions from our team were as follows: stop pulling the catheter when it comes to the extreme extent, no more than 20 times, and observation of catheter and mediastinum under the guide of DSA.

\section{Disclosure Statement}

The authors declare that there have no conflicts of interest to disclose.
Prof. Tianlei Cui

Division of Nephrology, Kidney Research Institute

West China Hospital, Sichuan University

Chengdu 610041 (China)

E-Mail tianleicui@163.com 


\section{References}

1 Forneris G, Savio D, Quaretti P, Fiorina I, Cecere P, Pozzato M, Trogolo M, Roccatello D: Dealing with stuck hemodialysis catheter: state of the art and tips for the nephrologist. J Nephrol 2014;27:619-625.

2 Hassan A, Khalifa M, Al-Akraa M, Lord R, Davenport A: Six cases of retained central venous haemodialysis access catheters. Nephrol Dial Transplant 2006;21:2005-2008.

3 Xiang DZ, Verbeken EK, Van Lommel AT, Stas M, De Wever I: Composition and formation of the sleeve enveloping a central venous catheter. J Vasc Surg 1998;28:260-271.

4 Wilson GJ, van Noesel MM, Hop WC, van de Ven C: The catheter is stuck: complications experienced during removal of a totally implantable venous access device. A single-center study in 200 children. J Pediatr Surg 2006; 41:1694-1698.

5 Hong JH: A breakthrough technique for the removal of a hemodialysis catheter stuck in the central vein: endoluminal balloon dilatation of the stuck catheter. J Vasc Access 2011; 12:381-384.

6 Carrillo RG, Garisto JD, Salman L, Merrill D, Asif A: A novel technique for tethered dialysis catheter removal using the laser sheath. Semin Dial 2009;22:688-691.

7 Huang SC, Tsai MS, Lai HS: A new technique to remove a "stuck" totally implantable ve- nous access catheter. J Pediatr Surg 2009;44: 1465-1467.

8 Hong JH: An easy technique for the removal of a hemodialysis catheter stuck in central veins. J Vasc Access 2010;11:59-62.

9 Cui T, Zhao Q, Zhou L, Li X, Fu P: A case report of a direct catheterization of tunneled cuffed catheter via superior vena cava: a choice after vascular access exhaustion. Blood Purif 2015;40:79-83.

10 Foley PT, Carter RM, Uberoi R: Endovascular removal of long-term hemodialysis catheters. Cardiovasc Intervent Radiol 2007;30:10791081. 